\title{
Differentiated Thyroid Carcinoma in Cambodia: A Single-Center Experience
}

\author{
Preap Ley \\ Department of Surgery, Sihanouk Hospital Center of Hope, Phnom Penh, Cambodia
}

\begin{abstract}
Objective: The goal of our study was to identify the prevalence of differentiated thyroid cancer and the results of surgical and postoperative hormone treatment of patients with thyroid cancer at Sihanouk Hospital Center of HOPE (SHCH) in Cambodia.

Methods: We retrospectively collected data of all patients diagnosed with thyroid carcinoma from 2005 to 2014 in the surgical department of $\mathrm{SHCH}$. The results of preoperative clinical assessment, laboratory tests, ultrasound, and fine needle aspiration cytology (FNAC) were correlated with the final histological findings.

Results: SHCH operated 587 cases of thyroid gland. Among those patients 36 cases (6.13\%) are diagnosed as thyroid cancer managed by surgery and thyroid stimulating hormone (TSH) hormonal suppressed treatments postoperatively. Among the 36 patients with differentiated thyroid carcinoma (DTC), 35 (97.23\%) were females and one (2.77\%) was male. The median age is 44 years old. Patients living in Phnom Penh are in high prevalence. Papillary carcinoma was the most prevalent of histopathological type $(n=33$, $91.66 \%)$. We performed FNAC in 22 cases but only 11 patients (28.20\%) had positive results for cancer and they received total thyroidectomy. The remaining patients had lobectomy or lobectomy with isthmusectomy and subsequent total thyroidectomy. All 36 patients have been taking L-thyroxine for TSH suppression, and they are still alive and continue their follow-up every 3 months regularly.

Conclusion: We conclude that total thyroidectomy results in a lower risk for tumor recurrence and improved survival rates in DTC. It is clear that after initial surgery, whether or not radioiodine therapy is administered, we suggest L-thyroxine therapy to prevent hypothyroidism and to minimize potential TSH stimulation of tumor growth.
\end{abstract}

Keywords: Thyroid gland; Carcinoma; Thyroidectomy; L-thyroxine

\section{INTRODUCTION}

Thyroid cancer is the most common endocrine malignancy, and its worldwide incidence has been slowly increasing during the past decades. In most areas of the world, the incidence of thyroid cancer among women is in the range 2-5/100,000; the incidence in men is 1-2/100,000. High-risk areas (incidence $>5 / 100,000$ in women) include Central America, Japan, and the Pacific Islands. International comparisons, however, are complicated by possible differences in diagnostic procedures. Survival from thyroid cancer is very good (over 85\% 5-year survival rate in Europe and North America), resulting in low mortality rates, below 1.2/100,000 in women and 0.6/100,000 in men in most parts of the world [1].

In 2008 in the United States, thyroid cancer was expected to have an annual incidence of 37,340 cases; today, it is the sixth most common cancer among women in that country. There has been a 2.4-fold increase in the incidence of thyroid cancer in the United States over the past 30 years, from 3.6/100,000 in 1973 to 8.7/ 100,000 in 2003 [2].

The fifteen Asian countries covered in this report (Japan, Taiwan, Singapore, Korea, Malaysia, Thailand, China, Philippines, Sri Lanka, Vietnam, Indonesia, Mongolia, India, Laos, and Cambodia) comprise $48 \%$ of the world's population ( 6.7 billion). All these countries show an increase of thyroid cancer prevalence, especially Japan and Indonesia [3]. In Cambodia, the prevalence of thyroid cancer remains unknown because a national cancer registry is not yet available. According to a 2005 report from Taiwan, the annual number of new cases of thyroid cancer in Cambodia 
was 76 among males and 141 among females. The mortality rates are the same for both sexes, around 0.9/100,000 of the age-standardized population [4].

There are many types of thyroid cancer, but the most common type by far is differentiated thyroid carcinoma (DTC), which comprises papillary, follicular, and Hurthle cell histological types derived from follicular epithelial cells, occurring in about $80 \%$ of cases. Although DTC typically has a good prognosis due to its long, indolent, and well-tolerated natural history, lifelong followup is recommended as late recurrence may occur after surgery and hormone suppression treatments [5].

The goal of our study was to identify the prevalence of thyroid cancer, especially DTC, and the results of surgical and postoperative hormone treatment of patients with thyroid cancer at our hospital in Cambodia.

\section{MATERIALS AND METHODS}

We retrospectively collected data of all patients diagnosed with thyroid carcinoma from 2005 to 2014 in the surgical department of Sihanouk Hospital Center of HOPE (SHCH). Patients were identified from pathology and surgical records. The results of preoperative clinical assessment, laboratory tests, ultrasound, and fine needle aspiration cytology (FNAC) were correlated with the final histological findings.

\section{Inclusion criteria}

The inclusion criteria in this study were patients with all DTCs including papillary, follicular, and Hurthle cell thyroid cancer who were operated and treated with hormonal drugs in our facility.

\section{Exclusion criteria}

The exclusion criteria were as follows: (1) patients with medullary, anaplastic, and other thyroid cancer; (2) patients operated outside of our hospital; (3) patients received hormone treatment outside of our hospital; and (4) patients with late- and advancedstage thyroid cancer and those referred outside of our hospital.

\section{Techniques}

At $\mathrm{SHCH}$, we perform a total thyroidectomy, with central lymph node or modified radical neck dissection if lymph node metastasis is confirmed, to achieve local disease control, irrespec- tive of patient age or tumor size. All patients undergo a similar procedure, as follows. We use a transverse Kocher skin incision of the neck with standard skin flaps, and also perform central neck or modified radical neck dissection while preserving the superior, inferior, and recurrent laryngeal nerves as well as parathyroid glands. In cases of poor hemostasis, a small Penrose or suction drain is placed for at least 24 hours postoperatively. Wound closure is performed using a variety of suture materials, according to individual preference, but we usually perform subticular closure. We never use antibiotic prophylaxis because thyroid surgery is a sterile procedure.

Operative specimens are sent to the pathology laboratory for verification of the diagnosis, and tumor-node-metastasis (TNM) stage, grading, resection margin, number of lymph nodes removed, and number of positive lymph nodes are recorded. During postoperative short-term follow-up visits, we document any exudation from the wound, collection of hematoma/seroma, drain situation, wound dehiscence, wound infection, voice change, and any hypocalcemia signs. All patients are treated with hormonal Lthyroxine postoperatively to reduce thyroid stimulating hormone (TSH) levels to $<0.1 \mu \mathrm{IU} / \mathrm{mL}$. We encourage patients who can afford radioiodine ablation therapy to go abroad and continue their treatment.

Long-term follow-up visits take place every 3 months. We see the patient to assess locoregional recurrences or distant metastases and regularly check their TSH levels. If needed, the L-thyroxine treatment dose is adjusted, to reach the goal of TSH $<0.1 \mu \mathrm{IU} / \mathrm{mL}$.

\section{RESULTS}

From January 2005 to December 2014, surgery was performed at $\mathrm{SHCH}$ in 587 cases of thyroid cancer. Among those patients, 36 (6.43\%) were diagnosed with DTC (34 patients had papillary and two had follicular thyroid cancer) and were managed with surgery and TSH hormone suppression treatment postoperatively. Among the 36 patients with DTC, 35 (97.23\%) were females and one (2.77\%) was male. Patient characteristics are summarized in Table 1. A total 36 patients aged $22-80$ years, with median age 44 years, were included in this study. Patients older than 60 years accounted for $11.76 \%$ of study participants, those aged $50-59$ years represented $11.76 \%$, those aged $40-49$ years represented $23.52 \%$, those aged 30-39 years represented $35.29 \%$, and $17.64 \%$ of patients were younger than 30 years old. We found a peak between ages 30 and 
Table 1. Clinical characteristics of 36 patients

\begin{tabular}{|c|c|}
\hline Variable & №. $(\%)$ \\
\hline \multicolumn{2}{|l|}{ Sex } \\
\hline Male & $1(2.7)$ \\
\hline Female & $35(97.3)$ \\
\hline Median age (yr) & 44 \\
\hline \multicolumn{2}{|l|}{ Age distribution (yr) } \\
\hline$<30$ & $7(17.6)$ \\
\hline 30-39 & $13(35.3)$ \\
\hline $40-49$ & $8(23.5)$ \\
\hline $50-59$ & $4(11.8)$ \\
\hline$>60$ & $4(11.8)$ \\
\hline \multicolumn{2}{|l|}{ Geographic distribution } \\
\hline Phnom Penh & $10(27.8)$ \\
\hline Prey Veng Province & $8(22.2)$ \\
\hline Kampong Cham & $6(16.7)$ \\
\hline Kandal & $6(16.7)$ \\
\hline Kompot & $2(5.5)$ \\
\hline Battambang & $2(5.5)$ \\
\hline Posath & $2(5.5)$ \\
\hline \multicolumn{2}{|l|}{ Location of cancer } \\
\hline Right lobe & $32(88.9)$ \\
\hline Left lobe & $4(11.1)$ \\
\hline \multicolumn{2}{|l|}{ Surgical procedure } \\
\hline Total thyroidectomy & $33(91.7)$ \\
\hline Total thyroidectomy with ipsilateral radical neck dissection & $2(5.5)$ \\
\hline $\begin{array}{l}\text { Total thyroidectomy with bilateral radical neck dissection, } \\
\text { tracheotomy }\end{array}$ & $1(2.8)$ \\
\hline \multicolumn{2}{|l|}{ Postoperative complications } \\
\hline No complication & $33(91.7)$ \\
\hline Permanent hypocalcemia & $1(2.8)$ \\
\hline Esophagus injury & $1(2.8)$ \\
\hline Recurrent laryngeal nerve injury caused permanent tracheotomy & $1(2.8)$ \\
\hline
\end{tabular}

49 years. With respect to geographic distribution, 10 patients (27.77\%) were from Phnom Penh, eight (22.22\%) from Prey Veng Province, six (16.66\%) from Kampong Cham, six (16.66\%) from Kandal, two (5.55\%) from Kompot, two (5.55\%) from Battambang, and two (5.55\%) from Posath.

Most patients had goiter (neck mass) as a first sign, between 2 months and 10 years before they sought medical care. Among them, 11 patients (30.55\%) also had a rapidly developing neck mass, seven patients (19.44\%) had hoarseness (voice change), 28 (7.77\%) patients had dysphasia, five patients (13.88\%) had weight loss, and all (100\%) patients had euthyroid nodular goiter (Fig. 1).

In addition to clinical signs, all patients with goiter underwent laboratory testing including for T3, T4, and TSH levels; neck ultrasound, and chest radiography including the neck. Electrocardiog-

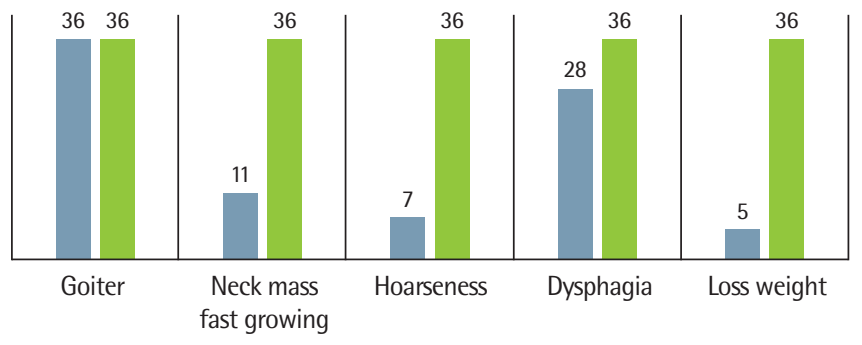

Fig. 1. Symptoms of thyroid cancer.

Table 2. Clinical characteristics and pathologic results

\begin{tabular}{lc}
\hline Variable & No. (\%) \\
\hline Location of cancer & $32(88.9)$ \\
Right lobe & $4(11.1)$ \\
Left lobe & \\
Surgical procedure & $33(91.7)$ \\
Total thyroidectomy & $2(5.5)$ \\
Total thyroidectomy with ipsilateral radical neck dissection & $1(2.8)$ \\
Total thyroidectomy with bilateral radical neck dissection, tracheotomy & \\
Postoperative complications & $33(91.7)$ \\
No complication & $1(2.8)$ \\
Permanent hypocalcemia & $1(2.8)$ \\
Esophagus injury & $1(2.8)$ \\
Recurrent laryngeal nerve injury caused permanent tracheotomy & \\
Type of cancer & $34(94.4)$ \\
Papillary & $2(5.6)$ \\
Follicular & \\
TNM (tumor-node-metastasis) classification & $5(13.9)$ \\
T2 N0 & $18(50.0)$ \\
T3 N0 & $3(8.3)$ \\
T3 N1 & $6(16.7)$ \\
T4 N1-3 & $4(11.1)$ \\
Unknown & \\
\hline
\end{tabular}

raphy was performed in patients older than 40 years old and those with suspected hyperthyroidism because of a high pulse rate. Among all patients with thyroid cancer, we performed FNAC in 22 cases but only 11 patients $(28.20 \%$ ) had positive results for cancer. In the remaining 28 patients, there were available results from the pathology department for samples obtained in the first surgery, thyroid lobectomy with isthmusectomy; these patients had a second operation, staged total thyroidectomy, within 1 week. Facilities for preparing frozen sections are not yet available at our hospital.

There were 32 patients (88.88\%) with cancer of the right lobe and only four cases (11.11\%) with left lobe involvement (Table 2). Two patients had right lateral neck lymph node metastases and one patient had bilateral neck lymph nodes metastases. We always 
perform total thyroidectomy in all patients with positive FNAC results (11 patients in this study). The remaining patients had lobectomy or lobectomy with isthmusectomy and subsequent total thyroidectomy. Among six patients with pT4 tumors, three had cancer cell-positive cytology not only with FNAC but also in the whole neck: one patient was pT4N3, and two were pT4N1a in the ipsilateral neck lymph nodes. We performed total thyroidectomy with ipsilateral modified radical dissection in the latter two cases and bilateral modified radical neck dissection with permanent tracheotomy in one patient who was N3 from the beginning because both recurrent laryngeal nerves were removed due to invasion by tumor.

Among all 36 patients who underwent surgery in our department, there was one case of permanent hypocalcemia and one case of pT4N3 with esophageal injury that we successfully repaired within 2 days. One patient with bilateral modified radical neck dissection also required permanent tracheotomy. The remaining 33 patients had no complications.

Papillary carcinoma was the most prevalent histopathological type (34 patients, 94.44\%). Only two patients (5.56\%) had follicular carcinoma. Five patients (13.88\%) were pT2N0, 18 patients (50.00\%) were pT3N0, three patients (8.33\%) were pT3N1, and six patients (16.66\%) were pT4N1-3; we did not know the TNM classification of the remaining four patients (11.11\%). At our hospital, patients who undergo total thyroidectomy remain in the hospital for only about 2-4 days; however, patients who undergo modified radical neck dissection remain hospitalized for 10-14 days.

All 36 patients in this study attended regular follow-up visits, which include the following. (1) The wound is checked every 3 days until suture removal, usually within 7-10 days. (2) All patients start L-thyroxine $100 \mu \mathrm{g}$ by mouth everyday in the early morning, at least 30 minutes before breakfast, from the first day postsurgery. (3) TSH levels are first checked 6 weeks later and hormone treatment is adjusted, to reach the goal of TSH $\leq 0.1 \mu \mathrm{IU} /$ $\mathrm{mL}$. Patients normally require 100 to $175 \mu \mathrm{g}$ /day L-thyroxine. (4) Long-term follow-up is conducted every 3 months (sometimes every 6 months), and L-thyroxine is provided according to clinical signs and TSH results. In patients with suspected local recurrence or neck lymph node metastasis, we always confirm using ultrasound. Chest, pelvic, or spinal radiographs are also repeated, according to patient complaints.

In the present study, one patient received radioiodine therapy in Singapore postoperatively. She is currently under treatment with
L-thyroxine at our hospital, to suppress TSH levels. In one patient with postoperative hypocalcemia, we provide calcium replacement therapy according to her blood calcium results. All 36 patients are still alive and receiving TSH hormone suppression treatment only, with regular follow-up visits every 3 months.

\section{DISCUSSION}

Weiss and Lado-Abeal [6] reported that thyroid nodules are common in the general adult population. In contrast, thyroid cancer is relatively rare and disease-specific mortality is low. The prevalence of palpable thyroid nodules in most series is 3\%-7\%. In our series, among 587 cases of thyroid gland surgery, the commonest first sign was thyroid nodule. A total 39 patients were diagnosed with thyroid cancer, and 36 of those patients had DTC. Patients with thyroid nodules should be referred to a specialist (endocrinologist or thyroid surgeon) for further assessment. Such a strategy speeds the process of reaching a diagnosis and is cost-effective. Clinical assessment by history-taking and examination usually does not lead to a precise diagnosis.

Mazzaferri [7] reported that although most people who develop thyroid cancer are middle-aged or older, papillary thyroid cancer can affect younger women, most commonly between the ages of 35 and 40 years. In addition, nearly every study shows that the patient's age at the time of diagnosis is an important prognostic variable and that thyroid carcinoma is more lethal after about age 40 years [7]. In our study, the median patient age was 44 years old, and we found an age peak between 30 and 49 years. Around 1,750 people develop thyroid cancer each year in the United Kingdom. Women are more commonly affected than men; some research has reported that thyroid cancer occurs about 3 times more often in women than in men [8]. However, in our series, we found that only one in 36 men were diagnosed with all thyroid cancers.

Thyroid association in the United Kingdom reports that the incidence of thyroid cancer is higher in patients with a long-standing thyroid nodular goiter or thyroiditis; in particular, solitary thyroid nodules have a 10\%-30\% risk of malignancy and this increases to 50\% in children [9]. All 36 (100\%) of our patients with DTC also had clinical signs of thyroid nodules (neck mass) for 2 months to 10 years. Among those, 11 patients (30.55\%) also had neck mass with a hard, irregular, fixed, and fast-growing character; other signs included hoarseness (19.44\%), dysphasia (7.77\%), and weight loss (13.88\%). 
Radiation is a well-recognized risk factor, such as radiation exposure from atomic fallout and radiotherapy to the neck. There is a genetic predisposition to thyroid cancer in families with the RET gene. The presence of this gene may result in family members with multiple endocrine neoplasia syndrome. Some reports also confirm other causes of thyroid cancer, including iodine deficiency, excess iodine in glands with pre-existing inflammation, and goitrogens like drugs and foods [10]. In our study, we could not identify any specific etiology or risk factors for thyroid cancer, but it is well known that many people in Cambodia have iodine deficiency and are exposed to many goitrogenic conditions.

Although physical examination, neck ultrasound, and other important lab tests (T3, T4, TSH) are the most common tools used to make a diagnosis of thyroid cancer, FNAC is the most effective. Modern use of FNAC of the thyroid gland was developed in Europe during the 1950s and 1960s. In the latter part of the 20th century, FNAC secured its rightful position as an essential first step in the assessment of a thyroid nodule. Thyroid FNAC is now well established as a first line diagnostic test in the evaluation of all thyroid swellings and is the single most effective test for preoperative assessment and diagnosis of thyroid nodules that are likely to be malignant and require surgery, when screening a large group of individuals with benign nodules that can be managed conservatively [11]. We performed FNAC in 22 of 39 patients with and without ultrasound, but only 11 cases of thyroid cancer were confirmed; this low positive rate may be owing to the experience and expertise of our surgeons.

In the literature, papillary thyroid carcinoma accounts for 70\%$90 \%$ of all thyroid malignancies, follicular cancers represent $15 \%-$ $20 \%$, medullary carcinoma accounts for $5 \%-10 \%$, and anaplastic carcinoma represents less than 5\%. Cancers classified as DTC include papillary, follicular, and Hurtle carcinoma. In our series, we found 34 patients with papillary, two patients with follicular, two patients with medullary, and one patient with anaplastic thyroid cancer. However, no patients with Hurthle cell thyroid cancer were found in this series.

Pasieka et al. [12] recommend total thyroidectomy for the routine treatment of tumors that are $1 \mathrm{~cm}$ or larger because approximately $50 \%$ of patients with cancer this size have additional cancer in the contralateral lobe. Most (80\%) papillary thyroid carcinomas are multifocal throughout the thyroid gland. All patients in this study had tumor sizes from T2 to T4 (from 1 to $4 \mathrm{~cm}$ ). Even tumors with smaller sizes can grow extensively beyond the thyroid gland, toward the spine or nearby large blood vessels. Ideally, we perform total thyroidectomy or subsequent total thyroidectomy. We also perform radical neck dissection if neck lymph nodes are positive for cancer.

The 2007 National Comprehensive Cancer Network guidelines report that the most common complications of thyroidectomy are hypoparathyroidism and recurrent laryngeal nerve injury, which occurs more frequently after total thyroidectomy. The rate of longterm recurrent laryngeal nerve injury and hypoparathyroidism after total thyroidectomy is $3 \%$ and $2.6 \%$, respectively. In our series, we had one patient with permanent hypoparathyroidism, one with recurrent laryngeal nerve injury caused by permanent tracheotomy, and one with esophageal injury.

Our patients had tumor sizes of at least T2 due to late presentation, and some patients were younger than 45 years old; we therefore treated these patients with total thyroidectomy. Following surgery, patients may be treated with high-dose radioiodine (131I) to address any occult distant metastases. Only one of our patients was able to afford this modality and received treatment in Singapore. However, all our patients received TSH suppression therapy with L-thyroxine, in the appropriate doses to reach TSH levels $\leq$ $0.1 \mu \mathrm{UI} / \mathrm{mL}$.

Thyroglobulin (a protein within the thyroid follicle) is a useful tumor cell marker for DTC and can be used to monitor patients following total thyroidectomy. Unfortunately, the thyroglobulin test is not available in our hospital. All our patients attend 3-monthly follow-up visits to check their clinical signs and TSH levels for the first 2 years after thyroidectomy, and every 6-12 months thereafter. Any abnormal physical findings can be evaluated with cervical ultrasonography; female patients usually undergo periodic cervical ultrasonography as an additional tool for surveillance and early detection of recurrent disease. A baseline chest radiograph is often performed and repeated every 2-3 years in patients who have undergone surgery for DTC.

\section{Conclusion}

Thyroid cancer, especially DTC, is the most common endocrine malignancy. DTC increases with age, and it is more common in women than men at our hospital. Although DTC typically has a good prognosis due to its long, indolent, and well-tolerated natural history, the primary treatment of DTC is surgical resection. For papillary thyroid cancer, most practitioners recommend a total thyroidectomy for any lesion over $1 \mathrm{~cm}$ in size. Because lesions 
smaller than $1 \mathrm{~cm}$ should not be subjected to biopsy, this would mean that all patients with this diagnosis should undergo total thyroidectomy. We conclude that total thyroidectomy results in a lower risk of tumor recurrence and improved survival rates.

We follow the recommended strategy in surgical oncology for patients with bulky disease. In particular, for patients with positive cervical lymph nodes palpated at presentation, a modified central neck dissection or modified radical neck dissection should be performed [13].

DTC responds to TSH by growing, producing and secreting thyroglobulin, and by trapping more iodine. This is why L-thyroxine therapy to suppress TSH decreases recurrence after surgery. When using the strategy of TSH suppression, it is important to follow plasma TSH levels, to ensure an adequate thyroxine dose. However, TSH suppression therapy can cause some complications such as anxiety, bone loss, and arrhythmias, which have to be considered, particularly in older patients. TSH suppression therapy is recommended for lowering TSH levels to at least $<0.1 \mu \mathrm{IU} / \mathrm{mL}$. After initial surgery, whether or not radioiodine therapy is administered, we suggest L-thyroxine therapy for all patients to prevent hypothyroidism and to minimize potential TSH stimulation of tumor growth. Careful long-term surveillance is also needed, which includes physical examination, thyroid hormone medication, and periodic laboratory and imaging studies.

\section{Recommendations}

Iodine deficiency causes goiter that is initially diffuse but eventually becomes nodular. It is known that the commonest first sign of thyroid cancer is thyroid nodule. Iodine deficiency is a global public health problem and achieving sufficient iodine nutrition in the population would eliminate this threat, specifically, through iodine supplementation of salt. Iodization of salt is the preferred method of increasing iodine intake in a community. Adding iodine during the packaging or processing of salt is an efficient means for distributing iodine on a mass basis, to prevent goiter. We should encourage people in Cambodia to use iodized salt.

Recent statistics indicate that Korea has an excellent treatment plan for thyroid cancer that is comparable to those of the United States, Japan, and European countries. Such a high survival rate is attributable to early diagnosis and detailed examination. By working together with experts from other countries, we can learn from their experience and achieve success in our own country.

In developed countries, advances in diagnostic modalities and pathologic analysis of thyroid cancer continue to evolve. In particular, high-resolution ultrasound should be available because it plays an increasingly important role in the management of thyroid cancer, including diagnosis of malignancy, preoperative lymphatic mapping, and postoperative surveillance. We should consider improving our ultrasound capability and skill, for effective screening and detection of thyroid cancers in our country.

Thyroid FNAC is now well established as a first line diagnostic test in the evaluation of all thyroid nodules; however, the skill of surgeons, radiologists, and pathologists in Cambodia is limited. These professionals need more training in this field, to properly assess patients with neck masses.

Surgery remains the mainstay of therapy; however, thyroid hormone TSH suppression and radioactive iodine ablation also contribute to treatment. We should consider making radioactive iodine ablation facilities available in our country in the near future.

We wish to emphasize that lifelong follow-up with TSH hormone suppression treatment is recommended, regardless of whether the patient receives radioactive iodine ablation, as late recurrence may occur after surgery.

\section{REFERENCES}

1. Boyle P, Levin B. World cancer report 2008. Geneva: World Health Organization; 2009.

2. Jemal A, Siegel R, Ward E, Hao Y, Xu J, Murray T, et al. Cancer statistics, 2008. CA Cancer J Clin 2008;58:71-96.

3. Health Grades Inc. Thyroid cancer [Internet]. Denver (CO): Health Grades Inc.; c2014 [cited 2018 May 10]. Available from: http://www.rightdiagnosis.com/t/thyroid_cancer/stats-country.htm.

4. The burden of cancer in Asia [Internet]. New York (NY): Pfizer Inc.; 2008 [cited 2018 May 10]. Available from: http://www.pfizer.com/files/products/cancer_in_asia.pdf.

5. Hundahl SA, Fleming ID, Fremgen AM, Menck HR. A National Cancer Data Base report on 53,856 cases of thyroid carcinoma treated in the U.S., 1985-1995 [see commetns]. Cancer 1998;83:2638-48.

6. Weiss RE, Lado-Abeal J. Thyroid nodules: diagnosis and therapy. Curr Opin Oncol 2002;14:46-52.

7. Mazzaferri EL. Thyroid papillary and follicular carcinoma. In: Mazzaferri EL, Samaan N, editors. Endocrine tumors. Cambridge: Blackwell Scientific Publications Inc.; 1993. p. 278-333.

8. Tidy C. Causes of cancer: what causes cancer? [Internet]. [place unknown]: Patient; c2018 [cited 2018 May 10]. Available from: http://www.patient. co.uk/health/what-causes-cancer.

9. British Thyroid Association, Royal College of Physicians. Guidelines for the management of thyroid cancer in adults. London: British Thyroid Association, Royal College of Physicians; 2007.

10. Livolsi VA, Asa SL. Endocrine pathology. Houston (TX): Gulf Professional Publishing; 2002.

11. Kocjan G. Fine needle aspiration cytology. Cytopathology 2003;14:307-8. 
12. Pasieka JL, Thompson NW, McLeod MK, Burney RE, Macha M. The incidence of bilateral well-differentiated thyroid cancer found at completion thyroidectomy. World J Surg 1992;16:711-6.
13. Poston GJ, Beauchamp RD, Ruers TJ. Textbook of surgical oncology. London: Informa Healthcare; 2007. 